\title{
Phase Shifters with Tunable Reflective Method Using Inductive Coupled Lines
}

\author{
Prof. Nitin Sherje \\ Professor, Department of Mechanical Engineering, S.K.N.C.O.E Pune India \\ npsherje@sinhgad.edu
}

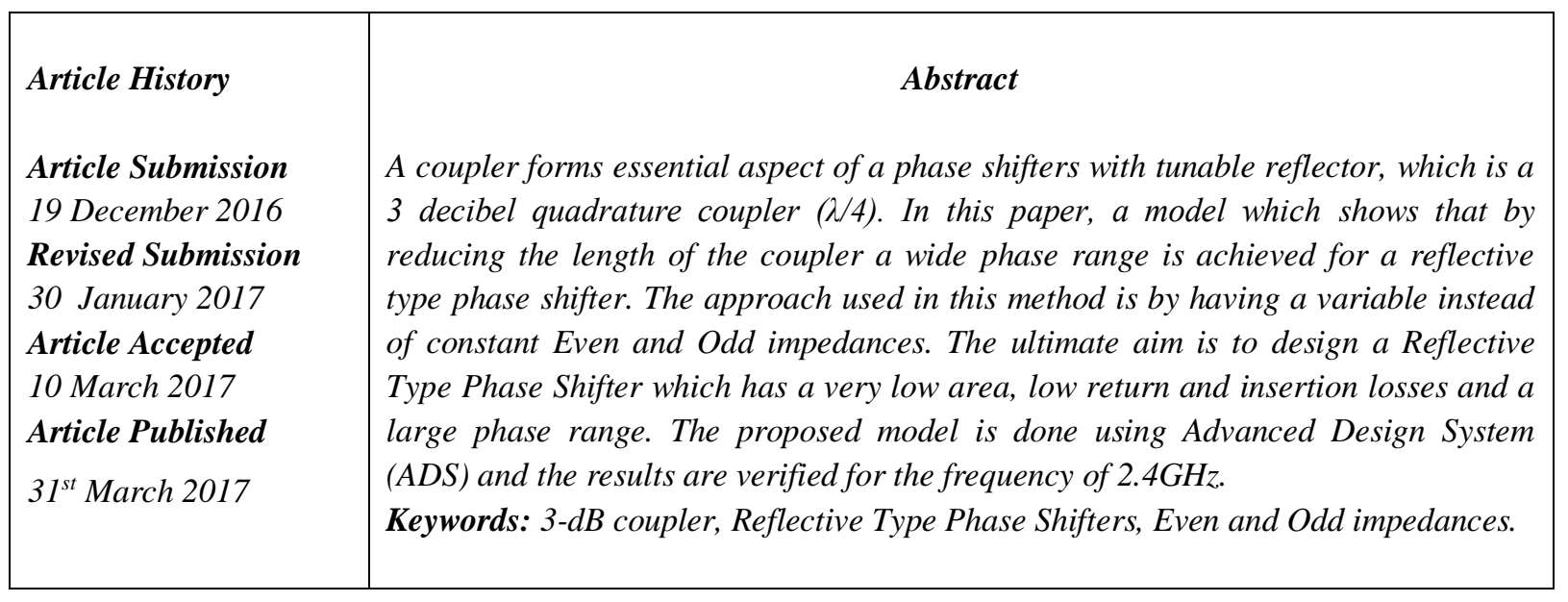

\section{Introduction}

Tunable phase shifters find its applications in many key areas such as frequency converters, MIMO, satellite systems, microwave instruments. Size of a coupler, insertion and return loss plays an optimum role in the phase shifter design especially reflective type [1][2]. The reason behind this is in MIMO a mobile handset is used which requires a small sized coupler instead of a larger one. Plus the cost also occupies an important role in this. But this reduction in size may affect the return loss of the coupler and hence the size should be reduced with a reasonable reduction in the return loss. Similarly insertion loss also, if a phase shifter is used in the transmitter part then the phase shifter may allow an undesirable power to the transmitting antenna and if the PS is used in the receiver part, then the SNR value of the signal may get degraded if the insertion loss is very high [3][4]. The 3-dB coupler is most commonly used in a RTPS which divides the entire signal into two orthogonal signals of equal energy. Then phase shifter is realized by placing impedance at two ports of the coupler except input and output port. The characteristic impedance is achieved using odd and even impedance by $\left(Z o *^{*} Z o e\right)=(Z o) 2$ A steady value is chosen for even and odd impedance and by using the above formula $50 \mathrm{ohms}$ is obtained. In this paper, a model which uses coupler length which is less than $(\lambda / 4)$ to reduce the area and by adjusting the impedance of coupler with wide phase range is obtained [5][6].

\section{Proposed Reflective Type Phase Shifters}

The RTPS has impedances connected at Coupled and Isolated ports. By variation of the impedances the phase of the signal at the output is changed. This is shown in figure 1.

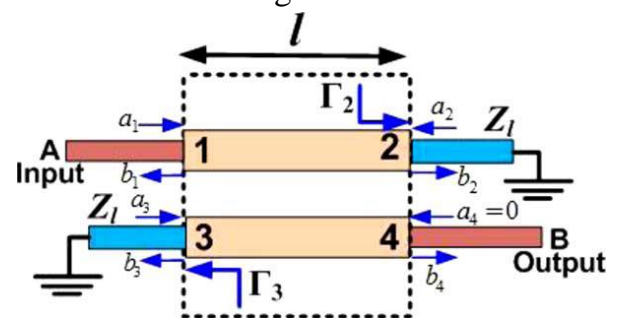

Fig 1: Proposed Reflective Type Phase Shifters 
Here the length 1 is variable, and to obtain a phase change the Load impedance $\mathrm{Zl}$ is changed. Here instead of using 3-dB coupler, a coupler of length less that 3-dB is used. Also the mode impedances such as Even and Odd are assumed to be independent variables, which mean they can take any value but square root of their product must be equal to the characteristic impedance [7][8].

The signals which are outgoing can be calculated for the incoming signal a using the formula

$$
\mathbf{b}=[\mathbf{I}-\mathbf{S} \Gamma]^{-1} * \mathbf{S a}
$$

where $\mathrm{S}=$ Scattering Parameters

$\Gamma=$ Reflection coefficient

$\mathrm{b}=$ vector of outgoing signals

$\mathrm{a}=$ vector of incoming signals

$\Gamma=$ Diagonal matrix of the reflection coefficient

$$
\Gamma 1=\Gamma 4=0 \quad \Gamma 2=\Gamma 3=\frac{(Z l-Z i n)}{(Z l+Z i n)}
$$

where Zin= Input impedance

$$
\begin{gathered}
Z \text { in }=Z o+\frac{2 *(Z i n e * Z i n o-Z o * Z o)}{Z i n o+Z i n e+2 Z o} \\
Z \text { ino }=Z o o * \frac{Z o+j Z o o * \tan (\beta l)}{Z o o+j Z o * \tan (\beta l)} \\
Z i n e=Z o e * \frac{Z o+j Z o e * \tan (\beta l)}{Z o e+j Z o * \tan (\beta l)}
\end{gathered}
$$

The S parameter calculation using odd and even impedances are as follows

$$
\begin{gathered}
S 11=\frac{(S 11 e+S 11 o)}{2} \\
S 21=\frac{(S 21 e+S 21 o)}{2} \\
S 31=\frac{(S 11 e-S 11 o)}{2} \\
S 11 e=\frac{j((Z o e / Z o)-(Z o / Z o e)) * \sin (\beta l)}{2 \cos (\beta l)+j(Z o e / Z o+Z o / Z o e) * \sin (\beta l)} \\
S 11 o=\frac{j(Z o o / Z o-Z o / Z o o) * \sin (\beta l)}{2 \cos (\beta l)+j(Z o o / Z o+Z o / Z o o) * \sin (\beta l)} \\
S 21 o=\frac{2}{2 \cos (\beta l)+j(Z o o / Z o+Z o / Z o o) * \sin (\beta l)}
\end{gathered}
$$


The important aspect that governs the routine of the phase shifter are $\phi, \mathrm{k}$ at the input terminal and output. For Fig1, they can be calculated as

$\mathrm{S}_{\mathrm{AA}}=\mathrm{S}_{\mathrm{BB}}=(\mathrm{b} 1 / \mathrm{a} 1) \quad \mathrm{S}_{\mathrm{BA}}=(\mathrm{b} 2 / \mathrm{a} 2) \quad \phi=\operatorname{angle}\left(\mathrm{S}_{\mathrm{BA}}\right)$

Assuming that the loads are connected at ports $2 \& 3$ then the varactor at these ports are varied such that $\mathrm{Cv}$ changes between maximum $(\mathrm{Cmax})$ and minimum $(\mathrm{Cmin})$ values and the phase range of the output signal is calculated using

$$
\Delta \phi=\phi(\text { when } \mathrm{Cv}=\mathrm{Cmin})--\phi(\text { when } \mathrm{Cv}=\mathrm{Cmax})
$$

as a function of coupler length and mode impedance. The varactor being a capacitor is assumed to have a capacitor ratio $\mathrm{Rc}=\mathrm{Cmax} / \mathrm{Cmin}$ to be 10 . Thus $\mathrm{Cmax}$ and $\mathrm{Cmin}$ are assumed to have values of $2 \mathrm{pF}$ and $0.2 \mathrm{pF}$. The capacitor is not assumed to be greater than 10 because of feasibility and hence instead of increasing the capacitor ratio an inductor is added in series to the varactor to get a higher phase shift. At the same time the inductor value should not exceed a threshold because even a higher inductance will reduce the phase range [9][10].

The odd impedance is assumed to have a low value and the even impedance a higher value to have a higher level of coupling between the coupling lines to get the desired phase. And also care should be taken such that the return loss does not come above $-10 \mathrm{~dB}$ and the reduction of the length of the coupler should be stopped if the return loss goes above $-10 \mathrm{~dB}$.

\section{ANALYSIS AND SIMULATION RESULTS}

The presented design is tested using phase shifters at $2.4 \mathrm{GHz}$ with $10 \mathrm{~dB}$ return loss. This frequency band is selected as it is used modern implementations including WLANs with MIMO front ends. This setup is controlled by tunable phase shifters.

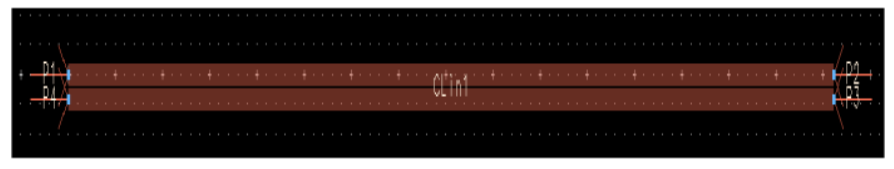

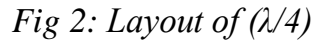

The proposed design has a precise setup that has small resistance value, moderate impedance values and small coupled structure with minimum $\mathrm{L}$ and $\mathrm{C}$ value that reduces stray capacitances. The required values are inductor $\mathrm{L}=6.5 \mathrm{nH}, \mathrm{Cmin}=0.2 \mathrm{pF}, \mathrm{Rc}=10, \mathrm{Zoo}=10 \Omega, \mathrm{Zoe}=225 \Omega$.

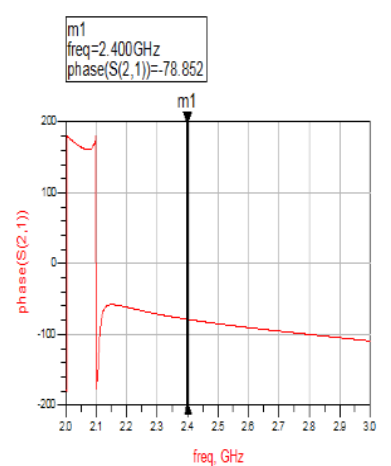

Fig 3: Simulated Results of phase for $C=0.2 p F$ and $2 p F(\lambda / 4)$ 


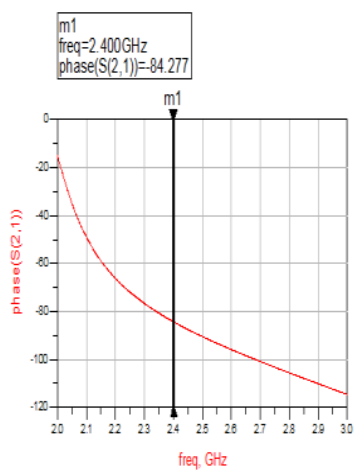

Fig 4: Simulated Results of phase for $C=0.5 p F$ and $2 p F(\lambda / 8)$

The presented design is tested using phase shifters at $2.4 \mathrm{GHz}$ with $10 \mathrm{~dB}$ return loss. This frequency band is selected as it is used modern implementations including WLANs with MIMO front ends. This setup is controlled by tunable phase shifters along with $\lambda / 8 P S$.

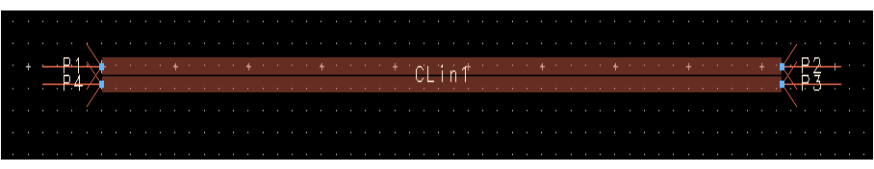

Fig 5: Layout for $(\lambda / 7)$

The proposed design has a precise setup that has small resistance value, moderate impedance values and small coupled structure with minimum $\mathrm{L}$ and $\mathrm{C}$ value that reduces stray capacitances. The required values are inductor $\mathrm{L}=6.5 \mathrm{nH}, \mathrm{Cmin}=0.2 \mathrm{pF}, \mathrm{Rc}=10, \mathrm{Zoo}=10 \Omega, \mathrm{Zoe}=225 \Omega$.

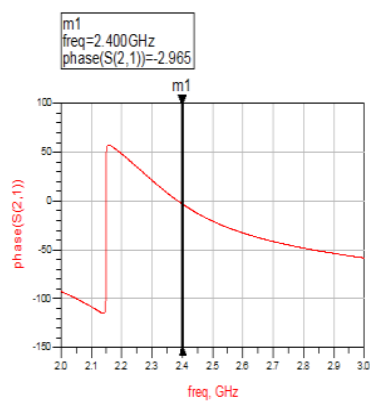

Fig 6: Simulated Phase for $C=2 p F$ and $0.2 P f(\lambda / 7)$

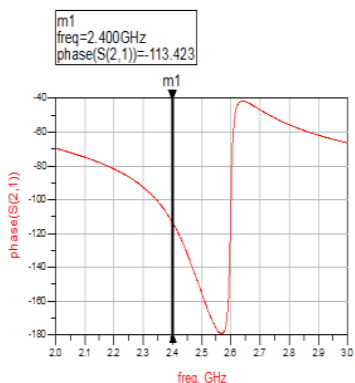

Fig 7: Simulated Phase for $C=5 p F$ and $0.8 P f(\lambda / 7)$ 
The simulated results are tabulated in table 1 and table 2 respectively.

Table 1: Coupler length Vs Phase range

\begin{tabular}{|c|c|c|}
\hline S NO & COUPLER LENGTH & PHASE RANGE(DEGREES) \\
\hline 1 & $\lambda / 4$ & 6 \\
\hline 3 & $\lambda / 7$ & -111 \\
\hline
\end{tabular}

Table 2: Coupler length Vs insertion loss and return loss

\begin{tabular}{|c|c|lc|lc|}
\hline S NO & COUPLER LENTH & \multicolumn{2}{|l|}{ INSERTION LOSS(dB) } & \multicolumn{2}{l|}{ RETURN LOSS(dB) } \\
\hline & & MIN & MAX & MIN MAX \\
\hline 1 & $\lambda / 4$ & 6.56 & 0.71 & 5.9 & 8.4 \\
\hline 3 & $\lambda / 7$ & 0.28 & 0.3 & 12.5 & 19.8 \\
\hline
\end{tabular}

\section{Conclusion}

Thus from the observation $3-\mathrm{dB}$ coupler which is of length $\lambda / 4$ gives a very less phase range compared to the lengths which are less than $3-\mathrm{dB}$ coupler. And the return loss value is also considerably around $10 \mathrm{~dB}$ for less lengths. Hence for the above values of Even and Odd impedance $(\lambda / 7)$ proves to be the better and optimum solution instead of $(\lambda / 4)$.

\section{References}

[1] Amin M. Abbosh, "Compact Tunable Reflection Phase Shifters Using Short Section of Coupled Lines”, IEEE Transactions on Microwave Theory and Techniques, Vol. 60, No. 8, August 2012.

[2] Alexander E. Martynyuk, Andrea G. Martinez-Lopez, and Jose I. Martinez Lopez,” 2-bit X-Band Reflective Waveguide Phase Shifter With BCB-Based Bias Circuits", IEEE Transactions on Microwave Theory and Techniques, Vol. 54, No. 12, December 2006.

[3] Hossein Zarei, Cameron T. Charlesand David J. Allstot, "Reflective-Type Phase Shifters for MultipleAntenna Transceivers, IEEE Transactions on Circuits and Systems-i: Regular Papers, Vol. 54, No. 8, August 2007.

[4] Yue Li, , Magdy F. Iskander, Zhijun Zhang, and Zhenghe Feng,"A New Low Cost Leaky Wave Coplanar Waveguide Continuous Transverse Stub Antenna Array Using Metamaterial-Based Phase Shifters for Beam Steering”, IEEE Transactions on Antennas and Propagation, Vol. 61, No. 7, july 2013.

[5] Frank Ellinger, Rolf Vogt,and Werner Bächtold, "Ultracompact Reflective-Type Phase Shifter MMIC at C-Band With 360 Phase-Control Range for Smart Antenna Combining”, IEEE Journal of Solid-State Circuits, Vol. 37, No. 4, April 2002

[6] Behzad Biglarbegian, Mohammad Reza Nezhad-Ahmadi ,Mohammad Fakharzadeh and Safieddin Safavi-Naeini," Millimeter-Wave Reflective-Type Phase Shifter in CMOS Technology", IEEE Microwave and Wireless Components Letters, Vol. 19, No. 9, September 2009.

[7] Hyukjin Kwon, Hongwook Lim, and Bongkoo Kang," Design of 6-18 GHz Wideband Phase Shifters Using Radial Stubs", IEEE Microwave and Wireless Components Letters, Vol. 17, No. 3, March 2007

[8] You Zheng, and Carlos E. Saavedra," An Ultra-Compact CMOS Variable Phase Shifter for 2.4-GHz ISM Applications", IEEE Transactions on Microwave Theory and Techniques, Vol. 56, No. 6, June 2008

[9] Y. Huang, C. Lin and T. Wu, "Balanced bandpass filter with intrinsic common mode suppression using slot coupled lines and microstrip loading lines," 2016 46th European Microwave Conference (EuMC), London, 2016, pp. 1171-1174, doi: 10.1109/EuMC.2016.7824557. 
[10] S. Kitanaka, K. Sakakibara and N. Kikuma, "Compact planar transmission-line transition directconnecting from a waveguide to four microstrip-lines," 2016 International Symposium on Antennas and Propagation (ISAP), Okinawa, 2016, pp. 376-377.

[11] S. Kagita, A. Basu and S. K. Koul, "Characterization of LTCC-Based Ferrite Tape in $\$\{X\} \$$-band and Its Application to Electrically Tunable Phase Shifter and Notch Filter," in IEEE Transactions on Magnetics, vol. 53, no. 1, pp. 1-8, Jan. 2017, Art no. 8000108, doi: 10.1109/TMAG.2016.2605078.

[12] X. Yang et al., "Compact and low loss phase shifter with low bias field using partially magnetized ferrite", IEEE Trans. Magn., vol. 49, no. 7, pp. 3882-3885, Jul. 2013.

[13] Ms. Sweta Minj. (2012). Design and Analysis of Class-E Power Amplifier for Wired \& Wireless Systems. International Journal of New Practices in Management and Engineering, 1(04), 07 - 13. Retrieved from http://ijnpme.org/index.php/IJNPME/article/view/9

[14] Prof. Naveen Jain. (2013). FPGA Implementation of Hardware Architecture for H264/AV Codec Standards. International Journal of New Practices in Management and Engineering, 2(01), $01-07$. Retrieved from http://ijnpme.org/index.php/IJNPME/article/view/11

[15] I. Viswanathan et al., "High performance compact microstripline phase shifter at C-band using yttrium iron garnet", IEEE Trans. Magn., vol. 45, no. 10, pp. 4176-4178, Oct. 2009.

[16] J. R. Bray and L. Roy, "Development of a millimeter-wave ferrite-filled antisymmetrically biased rectangular waveguide phase shifter embedded in low-temperature cofired ceramic", IEEE Trans. Microw. Theory Techn., vol. 52, no. 7, pp. 1732-1739, Jul. 2004 